\title{
Testing of the Possibilities of Using IMUs with Different Types of Movements
}

\author{
Pavol Kajánek \\ Slovak University of Technology, Faculty of Civil Engineering, Radlinského 11, 81368 \\ Bratislava, Slovakia, E-mail: pavol.kajanek@stuba.sk
}

\begin{abstract}
Inertial navigation system (INS) is a self-contained navigation technique. Its main purpose is to determinate the position and the trajectory of the object's movement in space. This technique is well represented not only as a supplementary method (GPS/INS integrated system) but as an autonomous system for navigation of vehicles and pedestrians, also. The aim of this paper is to design a test for low-cost inertial measurement units. The test results give us information about accuracy, which determine the possible use in indoor navigation or other applications. There are described some methods for processing the data obtained by inertial measurement units, which remove noise and improve accuracy of position and orientation.
\end{abstract}

Key words: Inertial Measurement Unit, Indoor Navigation.

\section{Inertial Measurement Unit}

Inertial measurement unit (IMU) is a complete three dimensional dead reckoning navigation system. It includes a set of inertial sensors such as accelerometers, gyroscopes and magnetometers (for absolute orientation). These sensors are comprised to one orthogonal system, which is known as body frame. Outputs of the gyroscope are angular rates, which are used for calculating attitude and outputs of the accelerometers are accelerations, which are used for the position determination ([1]).

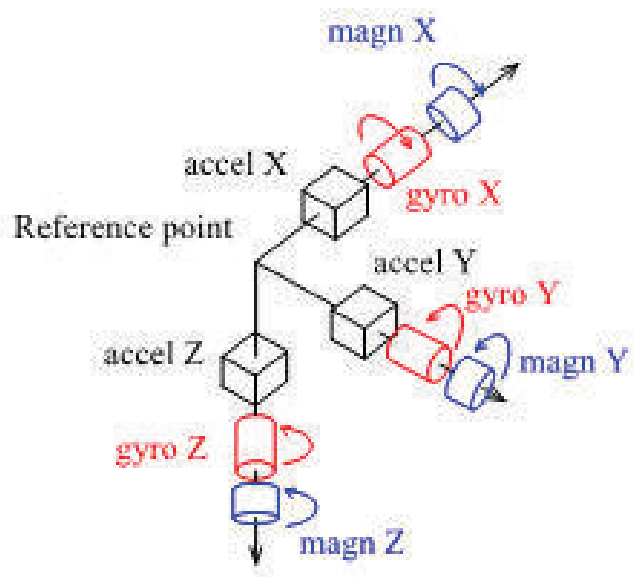

$$
\begin{aligned}
& \text { Legend: } \\
& \text { accel - accelerometer } \\
& \text { gyro - gyroscope } \\
& \text { magn - magnetometer }
\end{aligned}
$$

Figure 1: Body frame of inertial measurement unit

A gyroscope is an inertial sensor for measuring angular rate. For the strapdown IMU, three main types of gyroscopes are used, such as spinning mass gyroscope, optical gyroscope and vibratory gyroscope. Spinning mass gyroscope works on principle of conversation of angular momentum. Optical gyroscopes work on principle of Sagnac effect. Vibratory gyroscope operates on the principle of detecting Coriolis acceleration of vibrating element, when gyroscope is rotating ([3]). 
Accelerometer is based on the second Newton's law of motion to measure acceleration $\boldsymbol{a}$ by the measuring force $\mathbf{F}$, with the scaling constant $\mathbf{m}$ called proof mass ([3]).

$$
F=m \cdot a .
$$

For the strapdown systems the pendulous accelerometer and the vibrating-beam accelerometer are used. Pendulous accelerometer has proof mass, which is attached to a hinge, which deflects when acceleration occurs. Vibrating-beam accelerometer uses the piezoelectric effect. There is a pair of the quartz crystal beams mounted symmetrically. Each of beam vibrates on own resonant frequency. When the acceleration is induced along the sensitive axis, one beam is compressed while the other is stretched or tensioned. Acceleration is measured as difference in frequency. Magnetometer sensors works on the principle of Hall's effect ([3]). These sensors transform magnetic field to output voltage difference.

Accelerometer and gyroscope for the low-cost IMU are manufactured as micro-electromechanical sensors MEMS. MEMS are relatively small structures, which are manufactured using silicon or quartz. The advantages of MEMS sensors are small size, low weight, rugged construction, low power consumption, short start-up time, high reliability, low maintenance and compatibility with operation in hostile environments ([2]).

Currently, inertial measurement units have number of significant applications in surveying. IMU are used as a complementary method for navigation using GNSS, where the measurements made by inertial navigation system INS are used to interpolate the trajectory determined by using GNSS. Integrated GNSS / INS system is benefiting from the INS advantages such as high data rate and good short-term accuracy. IMU is often used for navigation of vehicles or pedestrian in indoor and outdoor environment.

\section{Mathematical Model of Data Processing}

Main aim of the navigation solution for a moving object (pedestrian or machine) is to determine its current position, velocity and orientation in reference coordinate system. In the following part of paper is described data processing of inertial measurements.

\subsection{Position Determination for a Moving Object}

This process can be divided into four steps. These are the attitude update, the transformation of specific force resolving axes, the velocity update and the position update. Approach of the processing of inertial measurements is shown in flowchart in Figure 2. The first step is numerical integration for the Euler angles (roll $\varphi$, pitch $\theta$ and yaw $\psi$ ) calculation from an angular rate, what is an output of gyroscopes. In the second step Euler angles are used to transform the acceleration $\boldsymbol{a}^{b}$ from body frame to acceleration $\boldsymbol{a}^{n}$ in navigation frame. The body frame changes its orientation in space with respect to a fixed navigation frame. Then we remove the force of gravity from the transformed acceleration.

$$
\begin{aligned}
& \varphi=\int_{t_{n-1}}^{t_{n}} \omega_{x}(t) d t, \quad \theta=\int_{t_{n-1}}^{t_{n}} \omega_{y}(t) d t, \quad \psi=\int_{t_{n-1}}^{t_{n}} \omega_{z}(t) d t \\
& \boldsymbol{a}^{n}=\boldsymbol{R}(\varphi, \theta, \psi) \cdot \boldsymbol{a}^{b}-\left[\begin{array}{lll}
0 & 0 & g
\end{array}\right],
\end{aligned}
$$

where $\mathbf{R}$ is current rotation matrix, $g$ is local gravity in yaw direction $\left(9,80665 \mathrm{~m} / \mathrm{s}^{2}\right)$. The next step is numerical integration of acceleration $\boldsymbol{a}^{n}$ and get the velocity $\mathbf{v}$. After second we use second numerical integration to calculate the position $\boldsymbol{p}$ from velocity.

$$
\boldsymbol{p}_{n}=\boldsymbol{p}_{n-1}+\iint_{t_{n-1}}^{t_{n}} \boldsymbol{a}_{n} d t
$$


where $\boldsymbol{p}_{n}$ is current position, $\boldsymbol{p}_{n-1}$ is previous position, $\boldsymbol{a}_{n}$ - is current acceleration in navigation frame, $\boldsymbol{v}_{n}$ is current velocity, t epoch of measurement. The mathematical model mentioned above is often called as dead-reckoning algorithm ([4]).

\subsection{Methods for Improving Position and Orientation Accuracy}

The disadvantage of the low-cost IMUs is low accuracy of the position determination that decreases with time of navigation. Low accuracy is caused by sensor errors that are cumulated by integration process.

There are numbers of methods, which could help improve accuracy such as:

- Allan variance method ([6]),

- Zero-velocity-update algorithm ZUPT used for foot mounted navigation ([5]),

- additional sensors: RFID, GPS, compass ([7]),

- specific information, which is obtained from maps: coordinates of door, direction of corridor and other ([8]).

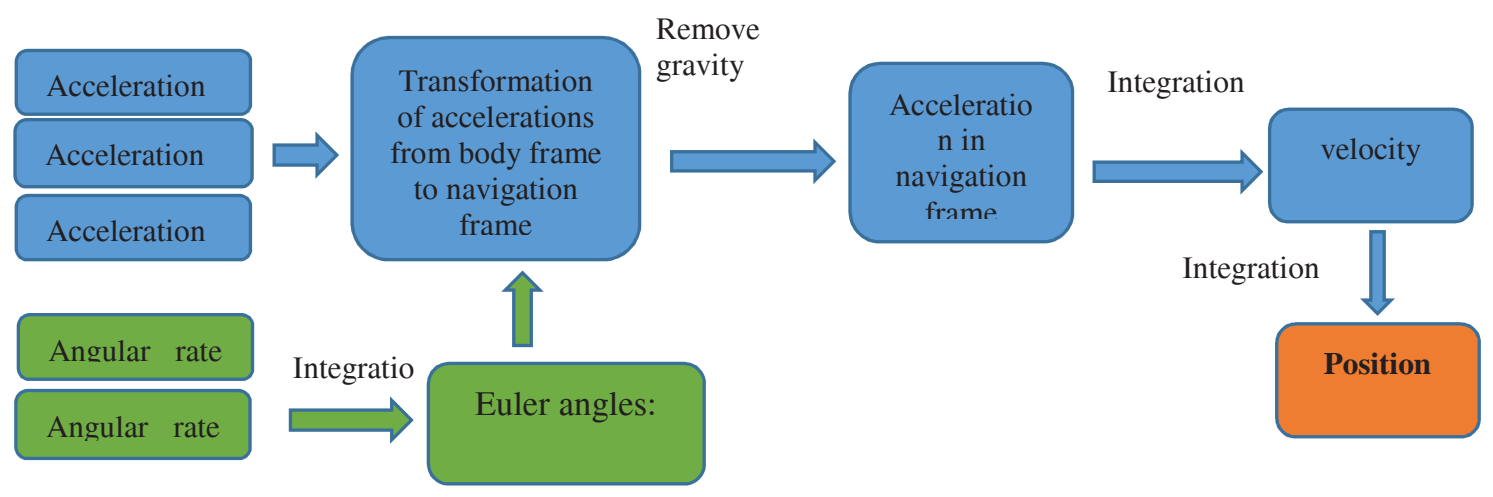

Angular rate

Figure 2: Flowchart of processing with inertial measurements

In these methods for purpose of suppression of noise components in the signal during the data processing the Kalman filter is often used ([1]).

\subsection{IMU Testing For Different Types of Movement with Accuracy Verification}

The application of sensors is strongly influenced by their accuracy, whereby the IMUs can be divided into several groups. The presented test concerns with testing low-cost IMUs, which due to low production costs, have lower accuracy compared to the IMUs designed for tactical applications. The designed tests are focused on the accuracy of the positioning using low-cost IMUs based on motion along a defined trajectory. Since the IMUs accuracy decreases with time of navigation, short-term linear movement will be performed. Further advanced movements of variable speed and orientation of motion (movement of pedestrians in the building) and also motion of IMU on testing cart (no vibrations with high amplitude caused by holding the IMUs in the hands of the pedestrian) can be used. Based on the proposed test two low-cost sensors (iNEMO STEVAL V2 MKI062V2 and IMU EEaS) will be tested.

The first test will be simulated simple and short-time movement of pedestrian. Tested IMUs will be positioned on the pedestrian's hand. There will be realized straight movement of testing device between points with known position (e.g. surveying pillars). Aim of the first test is testing of the accuracy of the IMUs in short-time term. 

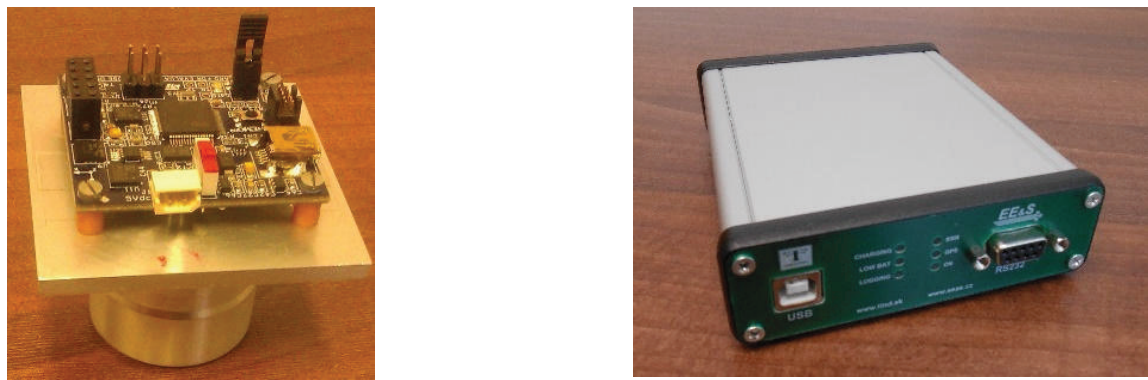

Figure 3: Tested devices: iNEMO STEVAL V2 MKI062V2 (left) and IMU EEaS (right)

In second test there will be simulated long-term advanced movement (standard pedestrian movement with random changes of acceleration and orientation). IMUs will be placed on the pedestrian's foot and pedestrian's hand. The aim of the second experiment is testing IMUs for indoor application, where the movement of pedestrians is more difficult (random changes of acceleration and orientation) and the inaccuracy grows with the traveled distance. Trajectory of movement will be limited by floor plan, where coordinated of passages (doors - red circle in Figure 4) are known.

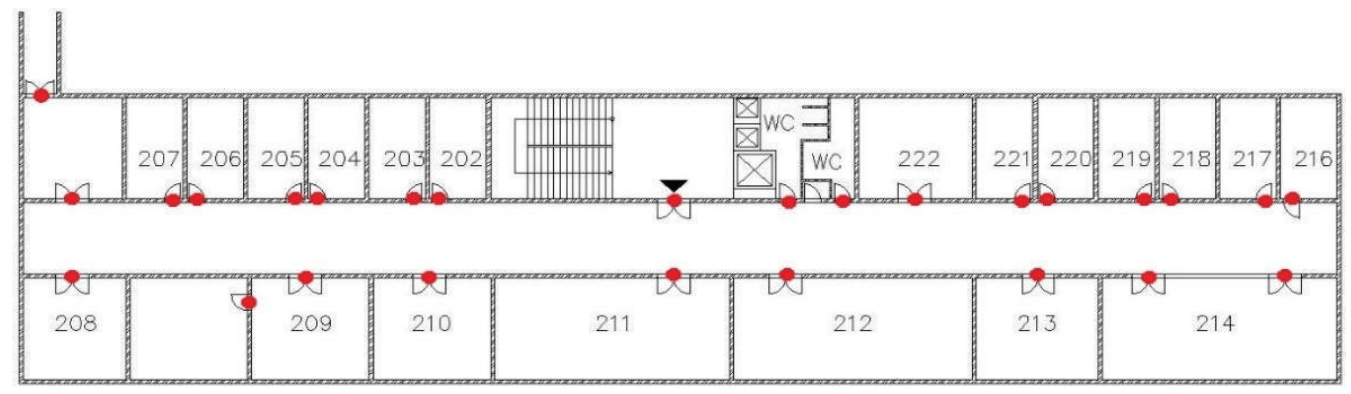

- Fixed point

Figure 4: Fix points as specific information in floor plan

In the third test IMUs will be placed on testing cart Figure 4. The purpose of this test is simulation of machine movement, where there are no vibrations with high amplitude caused by holding the IMUs in the hands of the pedestrian and the movement of the cart is more-les straight.

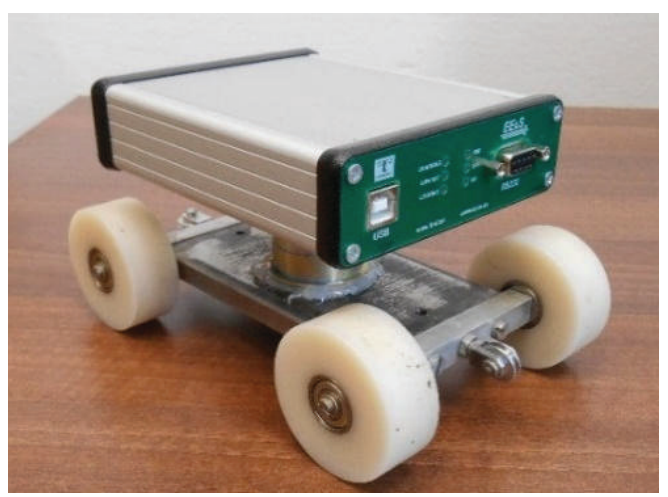

Figure 5: IMU EEaS placed on cart

\section{Design of Data Processing}

There will be used dead reckoning algorithm (chapter 3.1) to calculate position, velocity and attitude from output of IMUs. Unfortunately, there are errors in accelerometer and gyroscope measurements, which are cumulated in integration process. Random accelerometer and gyro noise 
have cumulative effect too. These facts cause, that the accuracy of the trajectory decrease significantly over the time.

An important part of the data processing will be the suppressing of the noise in the measurements. Before the integration process low pass filter will be used to suppress high-frequency components which contains the noise. Next step will be integration using Fast Fourier Transform (FFT). FFT allows transform of the measured signal from time domain to frequency domain. In frequency domain noise frequencies will be removed and after that inverse FFT will be used to convert signal back to the time domain. Then there will be used double numerical integration to calculate position from signal without noise ([9]).

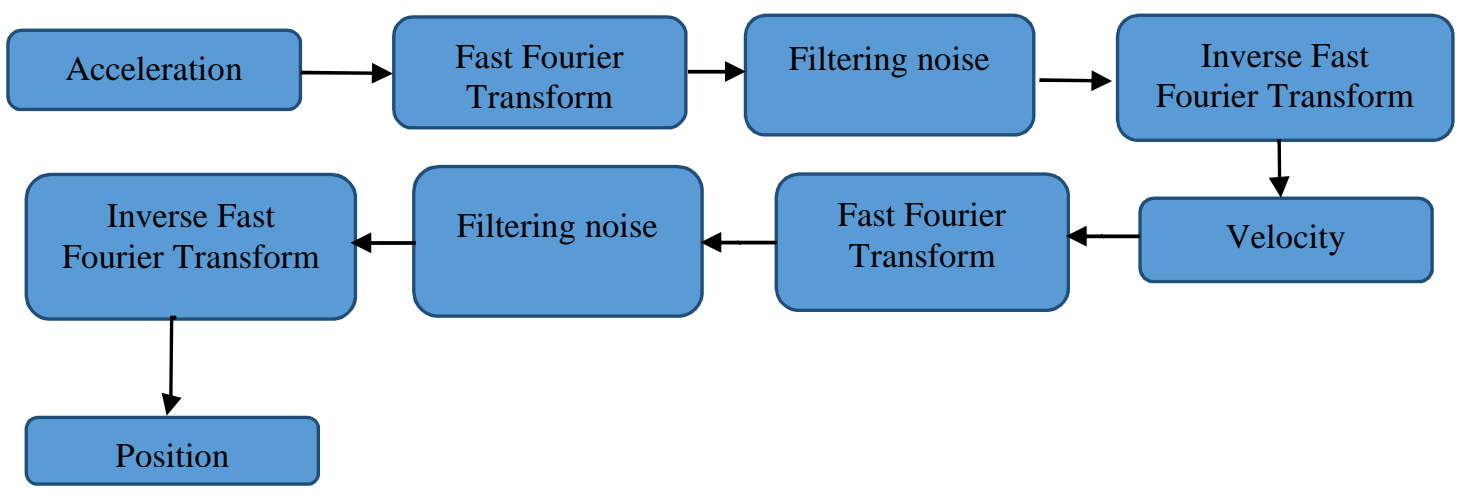

Figure 6: Scheme of integration with using Fast Fourier Transform

\section{Conclusion}

This paper describes exploitation of low-cost inertial measurement units for indoor applications. The main aim of the paper is to design test of IMUs for different types of movements (short-term linear movement, advanced long-term movement and movement on the testing cart), which represent typical situations in indoor navigation. The results of experiments give information about accuracy of tested IMUs, which is limiting factor for their possible application in pedestrian navigation, navigation of machines in buildings, etc. The paper also describes the data processing and methods, which could help to improve the accuracy of position and orientation. In future work we will test two IMUs (iNEMO STEVAL V2 MKI062V2 and IMU EEaS), where will use proposed test. Test result give informations about possibility of using tested IMUs in indoor navigation.

\section{Acknowledgement}

"This publication was supported by Competence Center for SMART Technologies for Electronics and Informatics Systems and Services, ITMS 26240220072, funded by the Research \& Development Operational Programme from the ERDF."

\section{References}

[1] GROVES, P.D. 2008. GNSS Technology and Aplications Series. Principles of GNSS, Inertial and Multisensor Integrated Navigation Systems. London: Artech House. 2008. 552 p. ISBN-13:978-1-58053-255-6

[2] FARREL J. A. 2008. AIDED NAVIGATION GPS with High Rate Sensors. USA: The McGraw-Hill Companies. 2008. 553 p. DOI: 10.1036/0071493298

[3] TITTERTON, D. 2004. Strapdown inertial navigation. Cornwall: MPK Books Limited, 2004. 510 p. ISBN 0-86341-358-7 
[4] FOXLIN E. Pedestrian tracking with shoe-mounted inertial sensors, IEEE Computer Graphics and Applications, no. December, pp. 38-46, 2005

[5] COLOMAR, S. D. Step-wise smoothing of ZUPT-aided INS. 2012. PhD Thesis. KTH.

[6] EL-SHEIMY, N., HOU, H. and NIU, X. Analysis and modeling of inertial sensors using Allan variance. In: Instrumentation and Measurement, IEEE Transactions , 2008, 57(1), p.140-149.

[7] RUIZ, A. R. J. et al. Pedestrian indoor navigation by aiding a foot-mounted IMU with RFID signal strength measurements. In: Indoor Positioning and Indoor Navigation (IPIN), 2010 International Conference on. IEEE, 2010. p. 1-7.

[8] JIMÉNEZ, A. R., et al. Improved Heuristic Drift Elimination (iHDE) for pedestrian navigation in complex buildings. In: Indoor Positioning and Indoor Navigation (IPIN), 2011 International Conference on. IEEE, 2011. p. 1-8.

[9] SLIFKA, L. An Accelerometer based approach to measuring displacement of a vehicle body. Master of Science in Engineering, Department of Electrical and Computer Engineering, University of Michigan-Dearborn, 2004. 